\title{
The long-term effects of the health coaching self- management program for nursing-home residents
}

\author{
This article was published in the following Dove Press journal: \\ Clinical Interventions in Aging \\ II July 2017 \\ Number of times this article has been viewed
}

\author{
Yeon-Hwan Park',2 \\ Sun-Hee Moon ${ }^{3}$ \\ Ji-Yeon $\mathrm{Ha}^{\prime}$ \\ Min-Hye Lee' \\ 'College of Nursing, Seoul National \\ University, ${ }^{2}$ The Research Institute \\ of Nursing Science, Seoul National \\ University, Seoul, ${ }^{3}$ Department of \\ Nursing, College of Health and \\ Welfare and Education, Gwangju \\ University, Gwangju, South Korea
}

Correspondence: Sun-Hee Moon Department of Nursing Science, College of Health and Welfare and Education, Gwangju University, 277 Hyodeok-Ro, Nam-Gu, Gwangju, South Korea $\mathrm{Tel}+82626702063$

Fax +8262670 2058

Email shmoon@gwangju.ac.kr
Background and aims: Little is known about whether a self-management program for nursing-home residents (NHR) with cognitive impairment is likely to have an impact on the care of this growing population. This study aimed to evaluate the effects of the health-coaching self-management program for NHR (HCSMP-NHR) on 1) self-efficacy and goal attainment scaling (GAS), 2) health status and quality of life (QoL) among older people, including those with cognitive impairment, in Korean nursing homes.

Methods: This was a cluster-randomized controlled trial. Participants in the intervention group ( $n=43$, mean age $=80.91 \pm 7.65$ years) received the HCSMP-NHR intervention, composed of group health education and individual coaching, for 8 weeks. Conventional care was provided to the conventional group $(n=47$, mean age $=80.19 \pm 7.53$ years $)$ during the same period. The effects of the HCSMP-NHR were measured three times: at baseline, week 9, and week 20.

Results: The intervention group showed better results for self-efficacy $(P=0.007)$, health distress $(P=0.007)$, depression $(P<0.001)$, and QoL $(P=0.04)$ at week 9. Mean GAS score of the intervention group gradually increased from -0.38 to 0.74 . The time $\times$ group interaction showed that the intervention group had significant improvements in QoL $(P=0.047)$, and significant reductions in health distress $(P=0.016)$ and depression $(P<0.001)$, while showing no deterioration in shortness of breath $(P<0.001)$.

Conclusion: Our study findings indicate that the HCSMP-NHR improved self-efficacy and GAS and enhanced the health status and QoL of NHR with chronic conditions who also had mild-to-moderate cognitive impairment. Moreover, these effects were successfully maintained over the 5 months of the trial. Further research is needed to establish the optimum intervention period and to assess the possibility of nationwide implementation of the HCSMP-NHR.

Keywords: health coaching, self-management program, nursing homes, long-term effects, cluster-randomized controlled trial

\section{Introduction}

Programs that allow older people with chronic illnesses to self-manage their symptoms and treatments based on lifestyle changes are essential for healthy living. ${ }^{1}$ Such self-management programs aim to improve skills in problem solving, exercise maintenance, medication use, and communication. ${ }^{2}$ According to previous studies, ${ }^{3,4}$ self-management programs can enhance both the physical and psychological health status of patients with chronic illnesses. One of these selfmanagement programs, the chronic disease self-management program (CDSMP) by Stanford University, has shown numerous beneficial effects, including decreasing blood pressure in patients with hypertension, reducing hemoglobin $\mathrm{A}_{1 \mathrm{c}}$ in patients with diabetes mellitus, and enhancing quality of life (QoL) in patients across different conditions. ${ }^{3,4}$ 
To date, it seems to have been difficult to apply selfmanagement programs to nursing-home residents (NHR). NHR require specifically designed self-management programs because most of them have health problems related to multimorbidity, thus they tend to be dependent on health care professionals (HCPs), and some of them have severe cognitive impairments. ${ }^{5}$ In this context, health coaching is seen as an effective strategy for older people to prevent worsening of chronic diseases and to enhance lifestyle modifications. ${ }^{6}$ The traditional approach for self-management strategies has depended on a top-down, one-way health education from HCPs to patients and their families, whereas health coaching elicits disease-management and lifestyle changes through reinforcement of the partnership between patients and HCPs. ${ }^{6,7}$ Health coaching could, therefore, be the best strategy to encourage, inspire, and empower older patients to reach their maximum potential. 6,8

Recently, a few studies have reported positive effects of a health-coaching self-management program for NHR (HCSMP-NHR), such as decreasing blood pressure in patients with hypertension, reducing depression and illness intrusiveness, and enhancing exercise behaviors and mental stress management. ${ }^{9-11}$ However, there were several limitations in the previous studies. First, the sample size in many of these studies was relatively small, and older people with cognitive impairments were excluded from the participants. ${ }^{10}$ In addition, the maintenance effects of the self-management program could not be confirmed. An ideal HCSMP-NHR should also be applicable to residents with moderate-tosevere cognitive impairment, who often live in long-term facilities, and the program should have its long-term effects measured. ${ }^{12}$

Therefore, the aim of the present study was to evaluate the effects of our program, the HCSMP-NHR, which was developed for older adults with cognitive impairment in nursing homes. The design of the HCSMP-NHR was based on a conceptual framework composed of a cascading process evaluation: self-efficacy and goal-setting, heath status, and
QoL (Figure 1). Health coaching is the practice of health education and health promotion within a coaching context., ${ }^{2,8}$ It could be an expected competency for HCPs to help older adults to improve their self-management skills. ${ }^{10}$ Coaching by HCPs may motivate older adults with chronic conditions to increase their self-efficacy and to take action toward making lifestyle changes through goal attainments. ${ }^{2,8,10}$ In addition, it could enhance the well-being of NHR and facilitate achievement of their best psychological and physical health status. ${ }^{9,10,13}$

This study's hypotheses were that, compared with participants assigned to conventional care, the participants in the HCSMP-NHR intervention would have 1) greater short-term improvement in self-efficacy and goal attainment scaling (GAS) scores and 2) greater short-term and long-term enhancement in health status and QoL.

\section{Methods \\ Study design}

The design of this study was a cluster-randomized controlled trial drawing a comparison between the HCSMP-NHR group and the conventional care group. The unit of randomization was the nursing home because the intervention could affect participant attitudes, self-efficacy, and behavioral changes, ${ }^{14}$ and because the residents were in constant contact with each other, these effects could diffuse from the intervention to the conventional care group if both groups were within the same home. We selected five nursing homes based on a number of factors, including size, history, and number of staff, to act as representative nursing homes in South Korea. The five nursing homes were allocated to the intervention group ( $\mathrm{n}=3$ ) or the conventional group $(\mathrm{n}=2)$ according to a computerized randomization method.

The HCSMP-NHR was provided to the intervention group for 8 weeks. It was composed of weekly group education for $40 \mathrm{~min}$ and individualized health coaching for $30 \mathrm{~min}$ per week. The conventional group received conventional care during the same period. The effects of the HCSMP-NHR

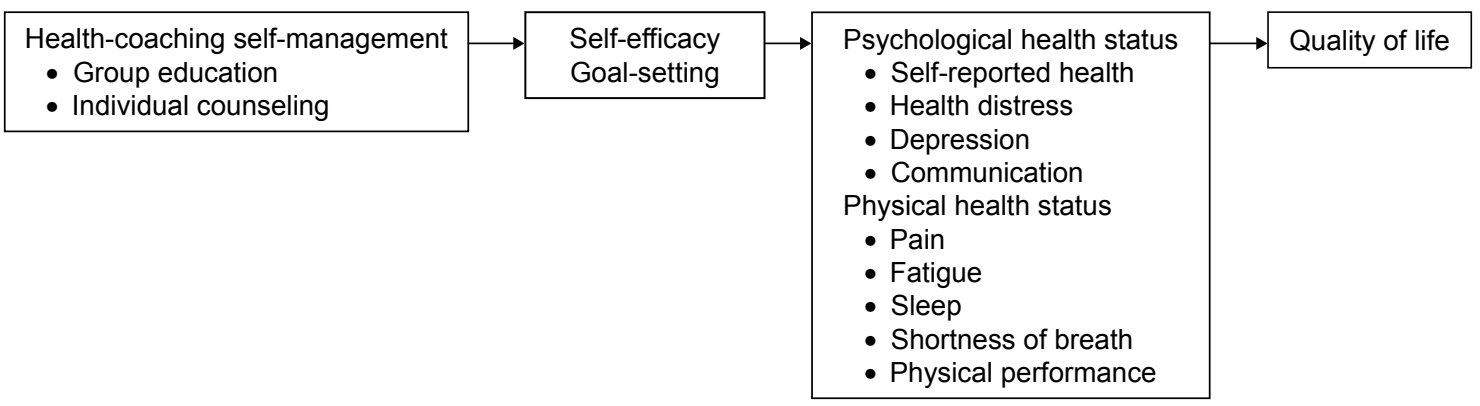

Figure I Framework of a health-coaching self-management program for nursing-home residents. 
on self-efficacy, GAS score, health status, and QoL were evaluated three times: at initiation (baseline), week 9, and week 20 .

\section{Participants}

In total, 110 older people residing in the five nursing homes were potential participants for the study. During the consent process, 12 residents were excluded because of deterioration in their health status, for example, if they had a lack of energy or had experienced a fall. We assessed the remaining 98 residents for study eligibility. The specific inclusion criteria were as follows: 1 ) age $\geq 65$ years; 2 ) ability to communicate; 3 ) diagnosis of one or more chronic diseases; and 4) the presence of mild-to-moderate cognitive impairment (based on scores of $\geq 10$ on the mini-mental state examination [MMSE]). The exclusion criteria for the study were 1) inability to attend group education or follow-up examination because the patient was an invalid or 2) discharge from the nursing homes during the program process. All 98 residents fitted the inclusion criteria and were allocated to the intervention group $(n=48)$ or the conventional group $(n=50)$, depending on their nursing home. In the intervention group, one participant missed the week 9 follow-up because of worsened dementia, whereas four participants missed the week 20 follow-up because of lack of energy, transfer to hospital, or discharge to home. In the conventional group, three participants missed the week 9 follow-up. Therefore, the final analysis assessed the data of 43 participants in the intervention group and 47 participants in the conventional group (Figure 2). The sample size for the analysis was sufficient to detect a small-to-moderate standardized effect size $(\mathrm{d}=0.4)$ using a two-tailed significance test with a power of $70 \%$ and an alpha level of 0.05 .

\section{Ethical considerations}

This study was approved by the Seoul National University institutional review board (IRB No 1502/001-015). A principal investigator explained the purpose and procedure of the study to the chief managers in the five selected nursing homes, and the chief managers recommended those among their NHR who fitted the inclusion criteria. The research team checked each resident's eligibility in detail and explained the purpose and specific procedure of the study to the potential participants. If the residents willingly decided to participate in the study, they signed a written informed consent form.

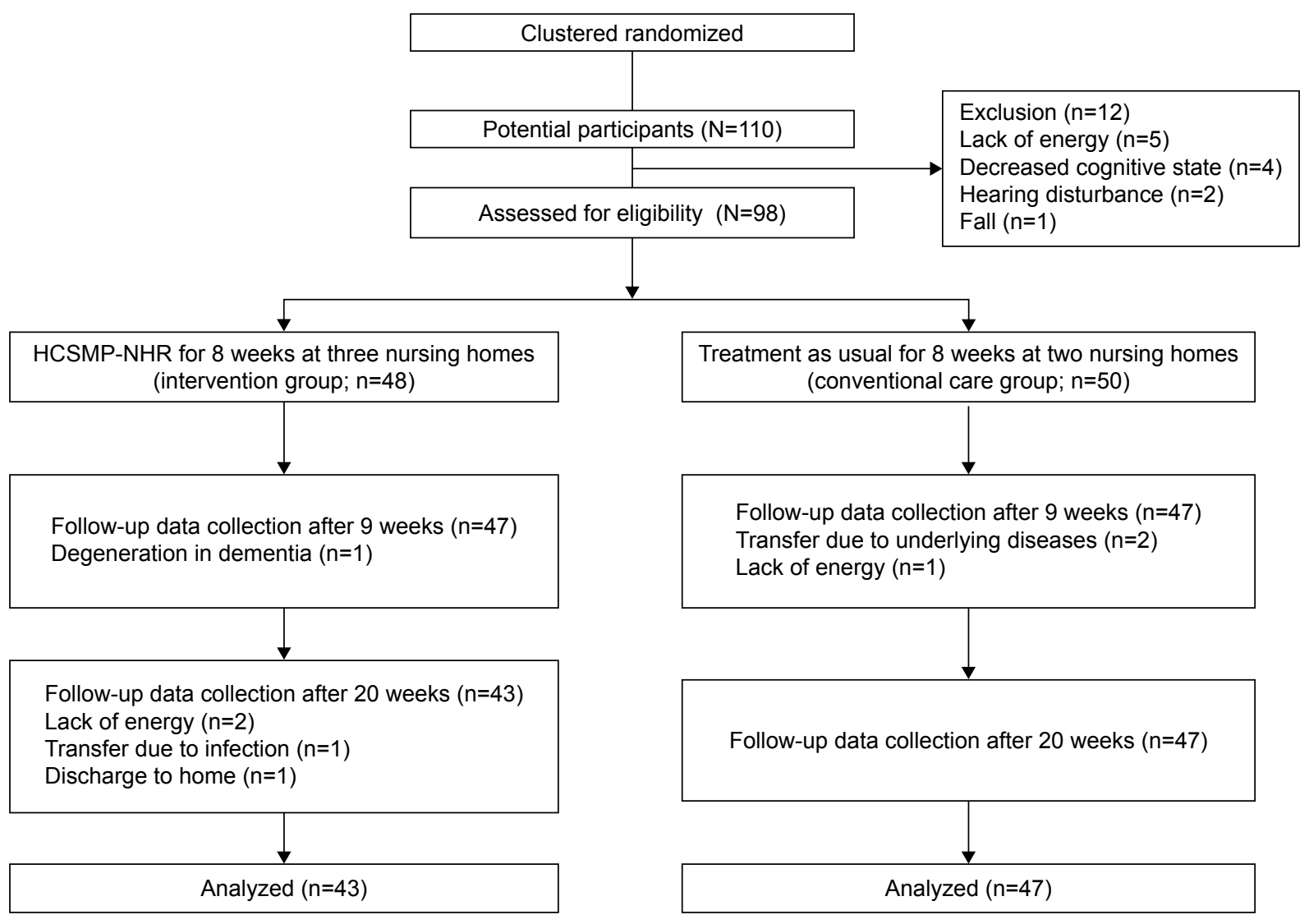

Figure 2 Flow chart for this study.

Abbreviation: HCSMP-NHR, health-coaching self-management program for nursing-home residents. 


\section{The HCSMP-NHR}

In developing the HCSMP-NHR, we focused on maximizing the effects of self-management depending on individual need and health status. The HCSMP-NHR comprised group health education and individualized health coaching for goalsetting. The contents of the HCSMP-NHR were developed and revised based on previous studies. ${ }^{9,10}$

\section{Training of the research team}

The principal investigator completed the leader training course for the CDSMP. We recruited six health coaches who were registered nurses to have at least 2-year clinical experience. To provide accurate health-coaching strategies based on the HCSMP-NHR, the research team prepared a standardized training course. Health coaches learned the principles and skills of health-coaching and self-management strategies under the guidance of the principal investigator for 1 hour/week for 2 months. During the individual health coaching, the six health coaches documented the coaching contents, then shared their experiences and opinions about health coaching, meeting once a week for competence equivalency as health coaches. In addition, the research team assessed the health-coaching process and gave feedback.

\section{Group health education}

The group health education focused on offering adequate knowledge, skills, and motivation for self-management to the residents. The contents of the group health education included essential topics for NHR: problem-solving, physical activity and regular exercise, healthy coping with daily stress, managing sleep, taking medication and managing side effects, communication skills, and goal attainment. Teaching materials suitable for NHR were prepared, including music, simple movements, and video clips.

The structured group health education was provided once a week for 8 weeks by six trained health coaches. Each session lasted $\sim 40 \mathrm{~min}$ and was carried out in a seminar room in the three nursing homes. The group education started with a review of previous topics and a short introduction, then went on to discussion of the residents' personal experience related to the topics, and was followed by a simple movement.

\section{Individualized health coaching}

After the group health education, health coaches provided one-to-one counseling to the participants in the intervention group. The individualized health coaching was based on partnership between participants and health coaches. ${ }^{6,7}$ Each session lasted 30 min for each participant and was carried out once in a week for 8 weeks. The health coaches began the session by assessing the participant's previous goal attainments, then new goals were set and the patient was offered counseling based on the group health education session, depending on individual capability and need. At the beginning of the intervention period, the participants selected goals and strategies from a list offered by the health coaches, but as time went on, some participants decided their weekly goals by themselves.

\section{Conventional group}

Participants in the conventional group maintained their usual lifestyle in the nursing homes for the 8 weeks that the program was carried out with the intervention group. After the follow-up evaluation at week 20 with the intervention group, the health coaches provided a condensed version of the individualized health-coaching program to the conventional group members in return for participation.

\section{Outcome measurements}

\section{The Stanford Research Instrument for Chronic Disease}

The Stanford Patient Education Research Center has developed tools to measure the effects of self-management programs for patients with chronic disease. ${ }^{15}$ In the current study, we used 31 items from the Stanford Research Instrument for Chronic Disease (SRICD). ${ }^{15}$

Self-efficacy in the study was measured by the SRICD, which is a widely used tool that has had its validity verified. ${ }^{15}$ The response scales for self-efficacy consisted of six items on the SRICD, with scores ranging from 1 (not at all confident) to 10 (totally confident), and the reliability was $0.91 .{ }^{15}$ Cronbach's $\alpha$ for self-efficacy in our study was 0.919 .

The psychological and physical health status of the participants were measured by 25 items on the SRICD: self-related health, health distress, Personal Health Questionnaire-8 (depression), communication with medical team members, energy, pain visual numeric, fatigue visual numeric, sleep visual numeric, and shortness of breath visual numeric. The reliability of tools for the health status on SRICD was $0.85-0.92 .{ }^{15}$ In our study, the Cronbach's $\alpha$ of health status items on SRICD was 0.618-0.933.

\section{Non-SRICD}

During the individualized health-coaching sessions with their participants, the health coaches recorded the goal attainments, new goals set, coaching time, specific strategies, 
and participants' response. Personal goals were related to the topics covered in the group education: exercise, stress management, communication, medication, and sleep. We assessed goal setting by GAS, a tool widely used in previous studies. ${ }^{10,16}$ GAS is constructed as a 5-point scale; 0 is the mid-point and reflects the goal that is set, while the 2 points above or below indicate exceeding or failing to meet that goal. So for instance, if the goal is that the participant is to engage in $30 \mathrm{~min}$ of exercise for 3 days/week, 0 means that they have attained this, while 1 point means they achieved 4-5 days of exercise and 2 points means $\geq 6$ days (exceeded the goal), whereas -1 point means $1-2$ days and -2 points means 0 days (failed to meet the goal). ${ }^{16}$

Physical health status was, additionally, measured by the short physical performance battery (SPPB) and sleep duration. The SPPB is composed of gait speed, chair stand, and balance tests, ${ }^{17}$ and the scores for these range from 0 (worst performance) to 12 (best performance). The reliability of the SPPB was $0.89^{17}$ and Cronbach's $\alpha$ in our study was 0.831 . In this study, we measured sleep duration by the hour.

We measured QoL using the EuroQoL five-dimension three-level questionnaire (EQ-5D-3L), which is a standardized tool to measure health-related QoL, developed by the EuroQoL Group. ${ }^{18}$ The response scales for the EQ-5D-3L consist of five items, with scores ranging from 1 (extreme problems) to 3 (no problems). The total score for the EQ5D-3L ranges from 3 (low QoL) to 15 (high QoL). The kappa value to evaluate the reliability of EQ-5D-3L was 0.32-0.64 and intra-class correlation coefficient for the Korean version was $0.61 .{ }^{19}$ In our study, Cronbach's $\alpha$ for the EQ-5D-3L was 0.64 .

\section{Data collection}

Data were collected from July 2015 to April 2016. Outcome variables were measured for the one-to-one interviews at baseline, week 9, and week 20. We recruited 10 senior nursing students and trained them in data collection.

\section{Statistical analysis}

Data were analyzed with SPSS (version 23.0; IBM SPSS, Armonk, NY, USA). We compared the baseline data of the two groups using the $t$-test for continuous variables and the chi-squared test for categorical variables. For hypotheses testing, paired $t$-test and repeated-measures ANOVA were used for comparing self-efficacy, health status, and QoL by time. Because of differences in baseline SPPB, we additionally analyzed the data using analysis of covariance with a Scheffé post hoc test.

\section{Results Baseline characteristics}

We completed the three sets of data collection for a total of 90 participants. The flow of participants through the trial is shown in Figure 2. The retention rate for the intervention group was $89.6 \%$. Most (78.9\%) of the 90 participants were women, and most of these (66.7\%) were widows (Table 1). Mean \pm SD age of the 90 participants was $80.53 \pm 7.55$ years (range 65-99). Baseline comparisons of the two groups before intervention did not differ except for the SPPB scores $(P=0.04)$.

\section{Comparison of outcomes after intervention}

The first hypothesis of the HCSMP-NHR was that participants in the intervention group would have greater short-term improvement in self-efficacy and in GAS scores than the participants assigned to conventional care. Self-efficacy in the intervention group using the HCSMP-NHR was significantly improved compared with the conventional group at week 9 $(P=0.007)$ (Table 2). Mean GAS score of the participants in the intervention group was -0.38 points at week 2 , but as the program proceeded, the score gradually increased, reaching 0.74 points at week 8 (Figure 3 ).

The second hypothesis of the study was that participants in the intervention group would have greater short-term and long-term improvement in health status and QoL than participants assigned to conventional care. In terms of the short-term outcomes, participants in the intervention group had significantly reduced health distress $(P=0.007)$ and depression $(P<0.001)$ and significantly better QoL $(P=0.04)$ compared with the conventional group at week 9. Participants in the intervention group did not show changes in shortness of breath $(P=0.16)$, whereas in the conventional group shortness of breath was increased at week $9(P=0.001)$.

For the long-term outcomes, differences from baseline to week 20 were compared, and interaction between groups by time was analyzed. At week 20, participants in the intervention group had significant reductions in health distress $(P=0.039)$, fatigue ( $P=0.013)$, and sleep problems $(P=0.016)$ compared with the conventional group. Participants in both the intervention and conventional groups had decreases in depression and pain at week 20. Participants in the intervention group did not have a change in energy $(P=0.281)$ or QoL $(P=0.276)$ at week 20, but participants in the conventional group had a deterioration in both energy $(P=0.009)$ and QoL $(P=0.021)$. For the SPPB, after control of baseline data, which was the covariate, there was no difference in SPPB between week 9 and week 20 (at week $9 P=0.782$; week $20 P=0.828$ ). 
Table I Baseline demographic and clinical characteristics and outcome variables for the HCSMP-NHR (N=90)

\begin{tabular}{|c|c|c|c|c|}
\hline Characteristics & $\begin{array}{l}\text { Intervention } \\
\text { group, } n=43(\%)\end{array}$ & $\begin{array}{l}\text { Conventional } \\
\text { group, } n=47 \text { (\%) }\end{array}$ & $\begin{array}{l}\text { Total, } \\
\mathbf{N}=90 \text { (\%) }\end{array}$ & $\begin{array}{l}\chi^{2} \text { or } \\
P \text {-value } \\
\end{array}$ \\
\hline \multicolumn{5}{|l|}{ Sex } \\
\hline Female & $32(74.4)$ & $39(83.0)$ & 7I (78.9) & 0.439 \\
\hline \multicolumn{5}{|l|}{ Marital status } \\
\hline Married & $15(34.9)$ & I3 (27.7) & $28(3 \mathrm{I} . \mathrm{I})$ & \multirow[t]{4}{*}{0.525} \\
\hline Separated & $0(0)$ & I (2.I) & I (I.I) & \\
\hline Widowed & $28(65.1)$ & $32(68.1)$ & $60(66.7)$ & \\
\hline Unmarried & $0(0)$ & I (2.I) & $\mathrm{I}(\mathrm{I} . \mathrm{I})$ & \\
\hline \multicolumn{5}{|l|}{ Long-term care grade } \\
\hline 1 & $0(0)$ & $2(4.3)$ & $2(2.2)$ & \multirow[t]{4}{*}{0.343} \\
\hline 2 & $13(30.2)$ & 14 (29.8) & $27(30.0)$ & \\
\hline 3 & $19(44.2)$ & $24(5 \mathrm{I} .1)$ & $43(47.8)$ & \\
\hline Unrated & II (25.6) & $7(14.9)$ & I8 (20.0) & \\
\hline \multicolumn{5}{|l|}{ Diagnosis } \\
\hline Diabetes mellitus & $15(34.9)$ & $16(34.0)$ & $31(34.4)$ & 0.93 \\
\hline Hypertension & $32(74.4)$ & $29(61.7)$ & $61(67.8)$ & 0.26 \\
\hline Arthritis & $12(27.9)$ & $8(17.0)$ & $20(22.2)$ & 0.31 \\
\hline Stroke & $13(30.2)$ & $20(42.6)$ & $33(36.7)$ & 0.276 \\
\hline Parkinson's disease & $2(4.7)$ & $5(10.6)$ & $7(7.8)$ & 0.438 \\
\hline \multirow[t]{2}{*}{ Dementia } & $20(46.5)$ & $16(34.0)$ & $36(40.0)$ & 0.283 \\
\hline & Mean \pm SD & Mean \pm SD & Mean \pm SD & $P$-value \\
\hline MMSE & $19.55 \pm 4.97$ & $18.68 \pm 5.98$ & $|9.1 \pm 5.5|$ & 0.454 \\
\hline Age (years) & $80.91 \pm 7.65$ & $80.19 \pm 7.53$ & $80.53 \pm 7.55$ & 0.656 \\
\hline Years of education & $7.46 \pm 5.11$ & $5.38 \pm 5.32$ & $6.38 \pm 5.30$ & 0.062 \\
\hline Length of stay in nursing home (months) & $22.63 \pm 23.28$ & $31.64 \pm 33.44$ & $27.33 \pm 29.23$ & 0.139 \\
\hline Number of chronic diseases & $3.65 \pm 1.49$ & $3.21 \pm 1.33$ & $3.42 \pm 1.42$ & 0.145 \\
\hline Variables & Mean \pm SD & Mean \pm SD & Mean \pm SD & $P$-value \\
\hline Six-item chronic disease self-efficacy & $31.88 \pm 15.55$ & $33.45 \pm 16.27$ & $32.7 \pm 15.86$ & 0.643 \\
\hline \multicolumn{5}{|l|}{ Psychological health status } \\
\hline Self-rated health & $3.56 \pm 1.47$ & $3.98 \pm 1.17$ & $3.78 \pm 1.33$ & 0.139 \\
\hline Health distress & $7.8 I \pm 6.04$ & $7.28 \pm 5.46$ & $7.53 \pm 5.72$ & 0.659 \\
\hline PHQ-8, depression & $6.72 \pm 4.50$ & $5.83 \pm 4.48$ & $6.26 \pm 4.49$ & 0.349 \\
\hline Communication with medical team members & $2.58 \pm 2.97$ & $2.72 \pm 2.32$ & $2.66 \pm 2.64$ & 0.8 \\
\hline \multicolumn{5}{|l|}{ Physical health status } \\
\hline Pain visual numeric & $3.16 \pm 3.44$ & $4.45 \pm 3.78$ & $3.83 \pm 3.66$ & 0.096 \\
\hline Fatigue visual numeric & $3.95 \pm 3.03$ & $4.7 \pm 3.49$ & $4.34 \pm 3.28$ & 0.283 \\
\hline Energy & $9.28 \pm 3.16$ & $9.53 \pm 3.70$ & $9.4 I \pm 3.43$ & 0.729 \\
\hline Sleep duration (hours) & $6.95 \pm 2.17$ & $7.34 \pm 1.72$ & $7.16 \pm 1.95$ & 0.35 \\
\hline Sleep visual numeric & $3.37 \pm 3.55$ & $3.77 \pm 3.40$ & $3.58 \pm 3.46$ & 0.592 \\
\hline Shortness of breath visual numeric & $1.33 \pm 2.50$ & $1.06 \pm 2.20$ & $1.19 \pm 2.34$ & 0.598 \\
\hline Short physical performance battery & $2.30 \pm 2.71$ & $1.21 \pm 2.18$ & $1.73 \pm 2.49$ & $0.04^{*}$ \\
\hline \multicolumn{5}{|l|}{ Quality of life } \\
\hline EQ-5D-3L & $10.40 \pm 2.27$ & $|0.04 \pm 2.3|$ & $10.21 \pm 2.29$ & 0.468 \\
\hline
\end{tabular}

Note: $* P<0.05$.

Abbreviations: HCSMP-NHR, health-coaching self-management program for nursing-home residents; MMSE, mini-mental state examination; PHQ-8, the eight-item patient health questionnaire depression scale; EQ-5D-3L, EuroQoL five-dimension three-level questionnaire.

The comparison between groups by time (baseline, week 9 , and week 20) showed significantly better outcomes in QoL $(P=0.047)$ and significantly greater reductions in health distress $(P=0.016)$ and depression $(P<0.001)$ for the intervention group. The analysis of shortness of breath by time showed that participants in the intervention group did not have a significant deterioration, whereas the conventional group $\operatorname{did}(P<0.001)$.

\section{Discussion}

The results of this study show that NHR with chronic conditions who participated in an 8-week program based on healthcoaching self-management achieved a significant improvement in self-efficacy and GAS and had enhanced health status and QoL, compared with NHR who received conventional care. Similar encouraging results have been reported, recently, in studies using coaching as a strategy for self-management. ${ }^{9,10,13}$ 


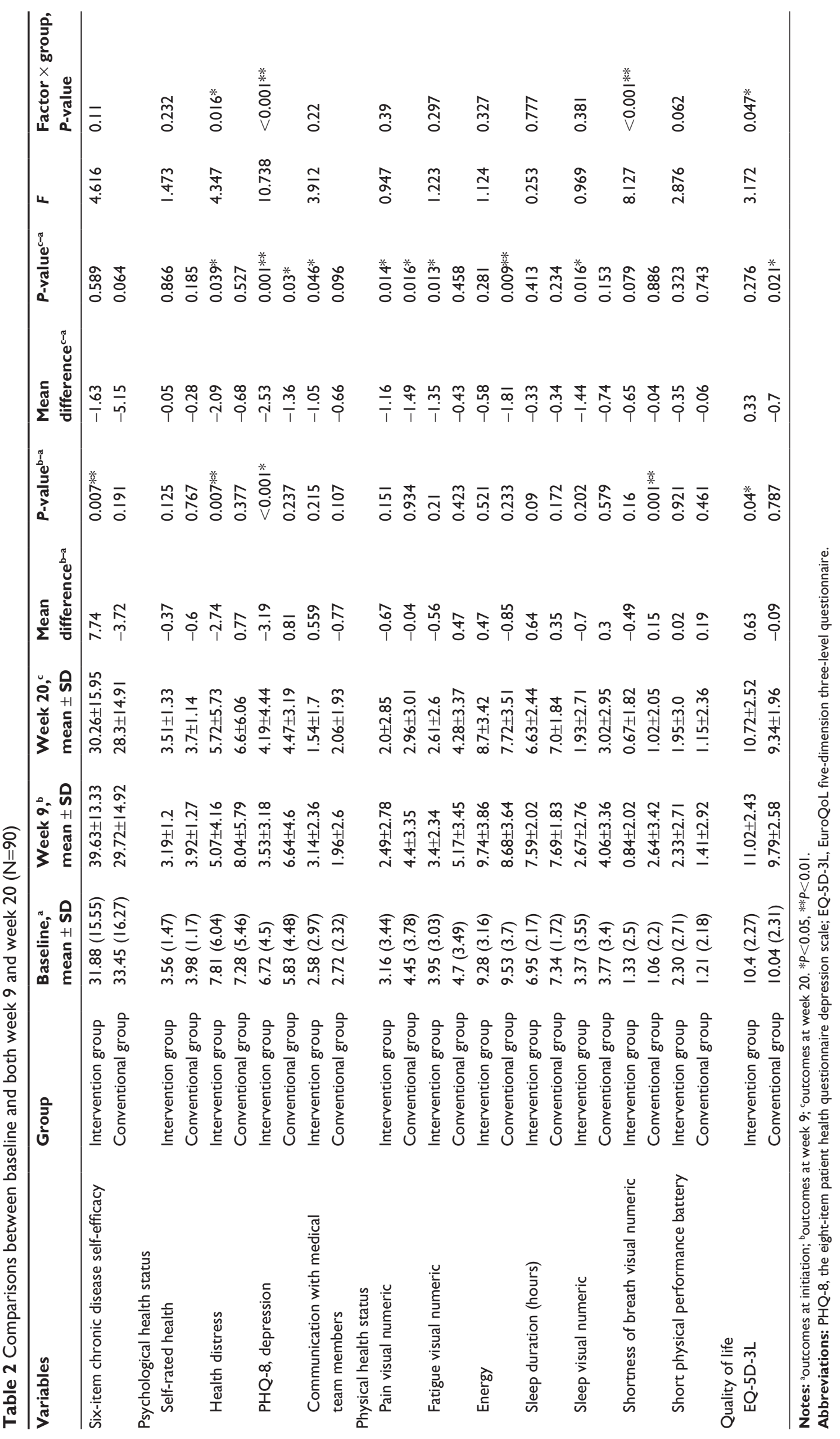




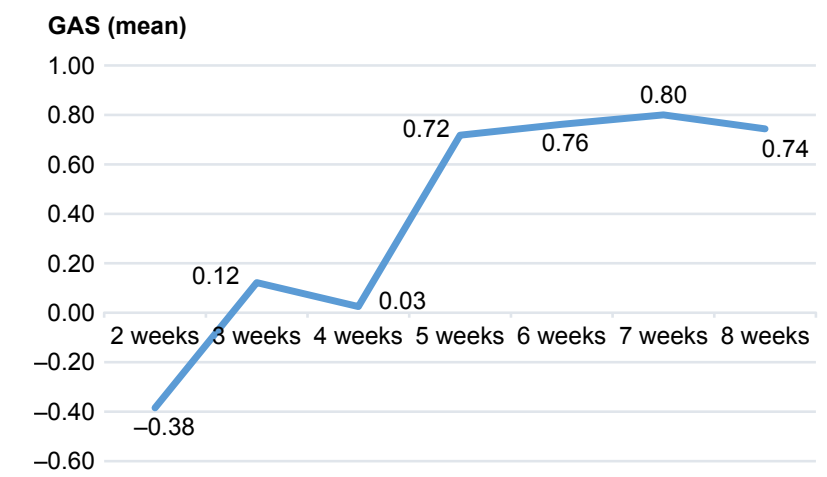

Figure 3 Variation in average goal attainment scaling (GAS) score in the intervention group over 8 weeks $(n=43)$.

The results of our study are in accord with previous studies for NHR, which showed that health-coaching interventions improved healthy behaviors, self-efficacy, and physical function, and also reduced depression. ${ }^{10,13}$ However, the present study expanded the target population for health-coaching intervention to cognitively impaired NHR, a growing population in long-term facilities. ${ }^{20}$ We developed the HCSMPNHR so it could be used for cognitively impaired NHR. Most of the existing self-management programs have been used only with community-dwelling older adults, ${ }^{3,21,22}$ and although self-management may be particularly needed for NHR, most cognitively impaired NHR are excluded from self-management programs. ${ }^{9,10}$ It is, therefore, particularly noteworthy that this program was successfully conducted with NHR who had mild-to-moderate cognitive impairment.

It should be noted that in the present study, the effects of the HCSMP-NHR were systematically analyzed based on a conceptual framework. In this study, the short-term outcomes were enhancement of self-efficacy through achievement of health goals. An interesting finding of this study was that GAS score gradually increased during the intervention. With regard to outcomes of a health-coaching intervention, GAS is a feasible and responsive parameter to measure achievement of health-related goals in long-term care. ${ }^{10,23}$ In previous studies, using GAS gave participants positive feedback because coaches encouraged participants to meet on a oneto-one basis and evaluated the personal goal achievement after individual coaching. ${ }^{10,24}$ This means that GAS used with the intervention group in our study acted both as an outcome measure and as an intervention strategy. GAS might have contributed to improving the interest and achievement of the participants in the intervention group. In our study, the healthy goals of exercise, stress management, communication, medication, and sleep were accomplished successfully by participants in the intervention group. Because the process of goal-setting can be one of the strategies for self-management, we could not assess the GAS score of participants in the conventional group, and therefore, we have to interpret the results related to GAS with caution. Future studies should consider corresponding measure and intervention measurements for residents assigned to conventional care.

The enhancement of self-efficacy in our results was consistent with the results of previous studies on older adults with multimorbidity, ${ }^{10}$ and support that a health-coaching intervention motivates residents' participation in intervention programs, resulting in a significant increase in residents' selfefficacy. In our study, self-efficacy was significantly increased at week 9, but this enhanced effect was not maintained until week 20. Self-efficacy in health-coaching self-management programs is a salient variable that affects health behavior. ${ }^{25,26}$ Self-efficacy is an important factor of self-management as a predictor of health behavior change and sustenance. ${ }^{27,28}$ Therefore, this result implies that additional interventions to improve self-efficacy are needed within 3 months. Another interpretation is that the intervention period using the HCSMP-NHR might be too short; the intervention period of self-management programs for community dwellers has been reported as varying from 7 to 12 weeks, ${ }^{29}$ while the duration for NHR is usually 8 weeks. ${ }^{9,10}$ Considering the impaired cognitive status of the NHR in the current study, a longer intervention period may be needed for lasting effects.

Both the short-term and long-term outcomes of our study, based on the conceptual framework, were improvement in health status and QoL. Outcome variables of self-management programs for NHR have mostly been compared pre- and postintervention in the short-term only, ${ }^{9,10}$ and only a few studies have measured long-term effects. ${ }^{13}$ Using the HCSMP-NHR and eliminating both group and time interaction, we found that health distress and depression (measures of psychological health state) were significantly decreased after the intervention, and shortness of breath (a measure of physiological health state) did not deteriorate. Chronic symptoms, such as fatigue, pain, shortness of breath, and health distress, are common and ingrained in NHR. ${ }^{30}$ In a previous study, it may have been difficult to check the change of these symptoms in a relatively short period. ${ }^{10} \mathrm{We}$ also identified that there were no changes in shortness of breath and energy in the intervention group following the health-coaching selfmanagement intervention, but there was deterioration in the conventional group. In particular, lack of energy, which is a common problem reported by older people, is a predictor of hospitalization in NHR. ${ }^{31}$ We consider that the preservation of the status quo in shortness of breath and energy in 
the intervention group might be a meaningful reflection of physical health status because aging and disease progress are associated with a decline in physical function. ${ }^{32}$

For both the intervention group and conventional group, the pain visual numeric scores were reduced at week 20 . This might be a possible effect of analgesics to decrease pain in the participants because medication is the usual method to control pain in patients. In our study, we could not exclude the effect of analgesics on pain, and hence we could not confirm that it was the health-coaching intervention that led to pain control in the intervention group. However, a recent study reported that a coaching intervention for people with dementia in nursing homes improved pain management. ${ }^{33}$ The effect of coaching interventions in pain management for nursing homes needs further evaluation.

The QoL scores of the intervention group in our study were significantly enhanced after the HCSMP-NHR at week 9 and week 20 compared with baseline. Unfortunately, we cannot confirm that this enhanced QoL will be maintained over the long term because self-efficacy in the intervention group had returned to the baseline by week 20 . As enhancement of self-efficacy in older adults is associated with improvement in QoL in the long-term, ${ }^{34} \mathrm{QoL}$ in this study could be reduced according to decreased self-efficacy in the long-term. Therefore, to design an optimum health-coaching program, additional strategies to improve self-efficacy and GAS in the long-term should be considered.

This study has some limitations. First, because we randomly assigned only five nursing homes, the results may not be generalizable to all of these institutions. Second, because the chief managers in the five nursing homes recommended potential participants, selection bias might have been created. To exclude such selection bias, it would be interesting to study paired sampling between the intervention and conventional groups. Third, considering the cognitive status of the participants, the intervention period was relatively short. To maintain the effects over the long-term, it may be necessary to have additional sessions or a longer intervention period. Fourth, during the period of the HCSMP-NHR, any competency differences between the six health coaches were not assessed. Partnership and active participation between health coaches and clients in dialogue and planning is an important attribute of this type of program. ${ }^{35}$ Although we had regular meetings, gave feedback after the first health-coaching session, and checked the coaching documents to minimize the competency differences between the health coaches, we cannot exclude that there may have been some differences in their competency levels.

\section{Conclusion}

On the basis of the current findings, we conclude that the HCSMP-NHR improved self-efficacy and GAS and enhanced the health status and QoL of NHR with chronic conditions who also had mild-to-moderate cognitive impairment. Therefore, the HCSMP-NHR might be a feasible intervention that may not only ameliorate the health status of NHR but also improve their QoL. The professional competence of HCPs will be needed to offer health coaching as a selfmanagement strategy in nursing homes. Further research is needed to establish the optimum intervention period and to consider additional interventions to maintain longer-term effects. The possibility of nationwide implementation of the HCSMP-NHR should also be assessed.

\section{Acknowledgments}

This study was supported by Basic Science Research Program through the National Research Foundation of Korea (NRF) funded by the Ministry of Education, Science and Technology (MEST) (grant number 2013R1A1A3A04008055).

\section{Disclosure}

The authors report no conflicts of interest in this work.

\section{References}

1. Richard AA, Shea K. Delineation of self-care and associated concepts. J Nurs Scholarsh. 2011;43(3):255-264.

2. Jordan JE, Briggs AM, Brand CA, Osborne RH. Enhancing patient engagement in chronic disease self-management support initiatives in Australia: the need for an integrated approach. Med J Aust. 2008; 189(10 Suppl):S9-S13.

3. Smith ML, Cho J, Ory MG, Salazar CI. Changes in quality of life indicators among chronic disease self-management program participants: an examination by race and ethnicity. Ethn Dis. 2013;23(2): $182-188$.

4. Chodosh J, Morton SC, Mojica W, et al. Meta-analysis: chronic disease self-management programs for older adults. Ann Intern Med. 2005;143(6):427-438.

5. Park YH, Bang HL, Kim GH, Ha JY. Facilitators and barriers to selfmanagement of nursing home residents: perspectives of health-care professionals in Korean nursing homes. Clin Interv Aging. 2015;10: $1617-1624$.

6. Huffman M. Health coaching: a new and exciting technique to enhance patient self-management and improve outcomes. Home Healthc Nurse. 2007;25(4):271-274; quiz 275-276.

7. Huffman MH. Health coaching: a fresh, new approach to improve quality outcomes and compliance for patients with chronic conditions. Home Healthc Nurse. 2009;27(8):490-496.

8. Hayes E, Kalmakis KA. From the sidelines: coaching as a nurse practitioner strategy for improving health outcomes. $J$ Am Acad Nurse Pract. 2007;19(11):555-562.

9. Park YH, Chang H, Kim J, Kwak JS. Patient-tailored self-management intervention for older adults with hypertension in a nursing home J Clin Nurs. 2013;22(5-6):710-722.

10. Park Y-H, Chang H. Effect of a health coaching self-management program for older adults with multimorbidity in nursing homes. Patient Prefer Adherence. 2014;8:959-970. 
11. Tse MM, Yeung SS, Lee PH, Ng S. Peer-assisted pain management program for nursing home residents: does it help to relieve chronic pain and enhance physical and psychological health. J Gerontol Geriat Res. 2015;S3:003.

12. Butterworth SW, Linden A, McClay W. Health coaching as an intervention in health management programs. Dis Manage Health Outcomes. 2007;15(5):299-307.

13. Frändin K, Grönstedt H, Helbostad J, et al. Long-term effects of individually tailored physical training and activity on physical function, well-being and cognition in Scandinavian nursing home residents: a randomized controlled trial. Gerontology. 2016;62(6):571-580.

14. Grimshaw JM, Eccles MP, Walker AE, Thomas RE. Changing physicians' behavior: what works and thoughts on getting more things to work. J Contin Educ Health Prof. 2002;22(4):237-243.

15. Standford Patient Education Research Center. Downloadable Research Instruments. Stanford Patient Education Research Center. Available from: http://patienteducation.stanford.edu/research/download.html. Accessed June 1, 2016

16. Becker H, Stuifbergen A, Taxis C, Beal CC, Pierini DM. The use of goal attainment scaling to facilitate and assess individualized change in a wellness intervention for women with fibromyalgia syndrome. J Holist Nurs. 2009;27(4):232-240.

17. Freire A, Guerra R, Alvarado B, Guralnik J, Zunzunegui M. Validity and reliability of the short physical performance battery in two diverse older adult populations in Quebec and Brazil. J Aging Health. 2012;24(5):863-878.

18. EuroQol Group. EuroQol - a new facility for the measurement of health-related quality of life. Health Policy. 1990;16(3):199-208.

19. Kim TH. Validity and Reliability Evaluation for EQ-5D in the General Population of South Korea. Ulsan: University of Ulsan; 2012.

20. Banaszak-Holl J, Fendrick AM, Foster NL, et al. Predicting nursing home admission: estimates from a 7-year follow-up of a nationally representative sample of older Americans. Alzheimer Dis Assoc Disord. 2004;18(2):83-89.

21. Barlow J, Wright C, Sheasby J, Turner A, Hainsworth J. Self-management approaches for people with chronic conditions: a review. Patient Educ Couns. 2002;48(2):177-187.

22. Ditewig JB, Blok H, Havers J, van Veenendaal H. Effectiveness of self-management interventions on mortality, hospital readmissions, chronic heart failure hospitalization rate and quality of life in patients with chronic heart failure: a systematic review. Patient Educ Couns. 2010;78(3):297-315.
23. Gordon JE, Powell C, Rockwood K. Goal attainment scaling as a measure of clinically important change in nursing-home patients. Age Ageing. 1999;28(3):275-281.

24. Yip AM, Gorman MC, Stadnyk K, Mills WG, MacPherson KM, Rockwood K. A standardized menu for goal attainment scaling in the care of frail elders. Gerontologist. 1998;38(6):735-742.

25. Jerant A, Moore M, Lorig K, Franks P. Perceived control moderated the self-efficacy-enhancing effects of a chronic illness self-management intervention. Chronic Illn. 2008;4(3):173-182.

26. King DK, Glasgow RE, Toobert DJ, et al. Self-efficacy, problem solving, and social-environmental support are associated with diabetes self-management behaviors. Diabetes Care. 2010;33(4):751-753.

27. Bandura A. Self-efficacy: toward a unifying theory of behavioral change. Psychol Rev. 1977;84(2):191-215.

28. Strecher VJ, DeVellis BM, Becker MH, Rosenstock IM. The role of self-efficacy in achieving health behavior change. Health Educ $Q$. 1986;13(1):73-92.

29. Crowe M, Jordan J, Burrell B, et al. Clinical effectiveness of transdiagnostic health management interventions for older people with multimorbidity: a quantitative systematic review. J Adv Nurs. 2016;72(10): 2315-2329.

30. Park MJ, Suh E, Lee JM. Nursing staffs' experiences of managing medical needs of the elderly in Korean long-term care facilities. Korean $J$ Adult Nurs. 2013;25(4):409-421.

31. Zengarini E, Hoogendijk EO, Perez-Zepeda MU, et al. Lack of energy and negative health-related outcomes in nursing home residents: results from the INCUR Study. J Am Med Dir Assoc. 2016;17(6):525-529.

32. Manini TM, Pahor M. Physical activity and maintaining physical function in older adults. Br J Sports Med. 2009;43(1):28-31.

33. Koppitz A, Bosshard G, Blanc G, Hediger H, Payne S, Volken T. Pain Intervention for people with Dementia in nursing homes (PID): study protocol for a quasi-experimental nurse intervention. BMC Palliat Care. 2017;16(1):27.

34. Elavsky S, McAuley E, Motl RW, et al. Physical activity enhances long-term quality of life in older adults: efficacy, esteem, and affective influences. Ann Behav Med. 2005;30(2):138-145.

35. Olsen JM. Health coaching: a concept analysis. Nurs Forum. 2014; 49(1):18-29.
Clinical Interventions in Aging

\section{Publish your work in this journal}

Clinical Interventions in Aging is an international, peer-reviewed journal focusing on evidence-based reports on the value or lack thereof of treatments intended to prevent or delay the onset of maladaptive correlates of aging in human beings. This journal is indexed on PubMed Central, MedLine,

\section{Dovepress}

CAS, Scopus and the Elsevier Bibliographic databases. The manuscript management system is completely online and includes a very quick and fair peer-review system, which is all easy to use. Visit http://www.dovepress. com/testimonials.php to read real quotes from published authors. 